\title{
EFFECTS OF TEMPERATURE ON IDENTIFIED CENTRAL NEURONS THAT CONTROL JUMPING IN THE GRASSHOPPER ${ }^{1}$
}

\author{
THOMAS W. ABRAMS ${ }^{2}$ AND KEIR G. PEARSON \\ Department of Zoology, University of Washington, Seattle, Washington 98195 and Department of Physiology, University of \\ Alberta, Edmonton, Alberta, T6G 2E1, Canada
}

Received August 28, 1981; Revised August 19, 1982; Accepted August 20, 1982

\begin{abstract}
Grasshoppers, like many poikilotherms, are generally more active at warmer body temperatures. In particular, they jump more frequently when warm. To determine the neuronal basis of this increase in jumping activity, we investigated the effects of temperature on the properties of identified central neurons known to be involved in the control of the jump; these included the fast extensor tibiae (FETi) motoneuron and the $\mathrm{C}, \mathrm{G}$, and $\mathrm{M}$ interneurons. Heating did not result in a reduction in the current or voltage threshold for action potentials; in most cases, there was an increase in the current threshold with heating.

At higher temperatures, the frequency-current relations of interneurons and motoneurons had steeper slopes. With strong current pulses, increasing the temperature resulted in an increase in the initial peak firing frequencies of central neurons and usually also in their steady state firing frequencies.

A second temperature effect favoring increased CNS activity in warm grasshoppers was increased afferent input from the periphery. In a broad variety of sensory receptors, there was a dramatic increase in their sensitivity to sensory stimuli at both threshold and suprathreshold intensities.

Various identified central neurons differed in the way in which some of their properties were influenced by temperature. The $\mathrm{C}$ and $\mathrm{G}$ interneurons showed a striking similarity in the unusual way in which their repetitive firing properties were influenced by heating. Since these neurons are sibling progeny of a single neuroblast, this shared physiological property is correlated with their developmental history.
\end{abstract}

Many poikilothermic animals, including grasshoppers, show increased behavioral activity at the higher body temperatures experienced during the course of normal daily temperature fluctuations (Laudien, 1973; Hussein, 1937; Chapman, 1969). A concomitant increase in the activity of central neurons with increasing temperature has been observed in several studies on a variety of species, including crayfish, cockroaches, slugs (Kerkut

\footnotetext{
${ }^{1}$ We are grateful to Dr. Corey Goodman for supplying us with Schistocerca nitens grasshoppers, for providing us with information about the lineage of the $\mathrm{C}$ and $\mathrm{G}$ neurons, and also for his useful discussion of our methods and results. We thank Drs. John Palka and Richard Levine for their critical reading of the manuscript and valuable suggestions and Dr. Barbara Graves for her helpful comments during the writing of the text. Dr. Richard Stein generously allowed us to use his PDP 11 computer for the analysis of spike trains.

${ }^{2}$ To whom correspondence should be addressed at his present address: Center for Neurobiology and Behavior, Columbia University and The New York State Psychiatric Institute, 722 West 168th Street, New York, NY 10032.
}

and Taylor, 1958), earthworms (Laverack, 1961), and crickets (Morrissey and Edwards, 1979). However, there has been little investigation of what temperature-dependent changes in cellular properties might account for this elevation of neuronal activity. Recently, Heitler et al. (1977) studied the effects of temperature on 2 identified neurons in acridid grasshoppers- the fast extensor tibiac motoneuron (FETi) and the dorsal unpaired median extensor tibiae neuron. These authors observed a decrease in the threshold for spike initiation with warming and suggested that this change in threshold might be a major mechanism for the increased neuronal activity in warm grasshoppers.

Our experiments on temperature effects were begun in conjunction with our research on a group of identified interneurons and motoneurons in the thoracic ganglia of grasshoppers that are involved in the control of jumping and kicking behavior (Pearson et al., 1980; Pearson and Robertson, 1981). In an attempt to increase the probability of kicking in our dissected preparations, we elevated their body temperature; if warm neurons had lower 
thresholds and therefore fired spikes more readily, then we expected these mounted grasshoppers to kick more often when they were heated above room temperature. This approach was unsuccessful. Experimental grasshoppers did not produce kicking responses reliably when warm, nor did the neurons that we were studying, including FETi, become more easily excited. Thus, the general increase in behavioral activity shown by the grasshoppers in our colony at higher temperatures was not correlated with a reduction in the spike threshold in the central neurons of experimental animals.

Subsequently, we went on to investigate the effects of temperalure on various other neuronal properties in an effort to identify the factors underlying the increased behavioral activity. It was an implicit assumption throughout this study that a change in the spike activity of at least some central neurons must accompany any temperature-dependent change in behavioral activity; more particularly, if a certain behavior occurs at a higher frequency, then at least the excitatory neurons which control that behavior must fire spikes more often. The jumping behavior of the grasshoppers in our colony shows a dramatic temperature dependence. When their temperature is raised from $21^{\circ}$ to $31^{\circ} \mathrm{C}$, these animals increase their frequency of spontaneous jumping by more than 5-fold (Abrams, 1982). We looked for changes with heating that would contribute to increased spike activity in the neurons known to be involved in jumping and kicking: the FETi motoneuron, which drives the rapid extension of the hindleg during the jump; the flexor tibiae motoneurons (Heitler and Burrows, 1977); and 2 interneurons that are important in coordinating the jump motor program, the $\mathrm{C}$ neuron and the $\mathrm{M}$ neuron (Pearson et al., 1980; Pearson and Robertson, 1981). The G neuron, an auditory interneuron with an excitatory input to the jump neural circuit (Kalmring, 1975; Pearson et al., 1980), also was studied extensively.

In this paper, we begin by presenting evidence that the threshold of central neurons is not reduced with heating. We then describe temperature effects on repetitive firing and synaptic transmission. Finally, we discuss experiments on the G neuron and on auditory receptors which indicate the significance of increased input from these afferents in determining how the G neuron's auditory response is affected by heating. In general, two effects of temperature stand out as potentially important in producing increased levels of activity in central neurons at warmer temperatures: (1) a steepening of the slopes of their frequency-current (f-I) relations and (2) an increase in the input to these central neurons from primary sensory neurons. The effect of temperature on a variety of sensory receptors is discussed in more detail in a second paper (T. W. Abrams and K. G. Pearson, manuscript in preparation).

\section{Materials and Methods}

\section{Animals and rearing}

The majority of experiments were done on adult $\mathrm{Lo}$ custa migratoria and Schistocerca americana gregaria grasshoppers from our colony at the University of Alberta. Grasshoppers were reared under crowded condi- tions with a 15:9 light-dark cycle; lighting was provided by a $40-\mathrm{W}$ incandescent bulb in each cage. During the dark phase, cage temperatures were $23^{\circ} \mathrm{C}$; during the light phase, the animals thermoregulated using the incandescent bulbs and generally maintained their body temperatures between $34^{\circ}$ and $36^{\circ} \mathrm{C}$. (Body temperatures were measured by inserting a small thermocouple into the thoraxes of the animals.) Resistance measurements, as well as some replications of experiments on thresholds and sensory neurons, were done on $S$. americana from a similar colony at the University of Washington. A small number of experiments also were carried out on Schistocerca nitens from a culture at Stanford University kindly supplied by C. Goodman. These animals were kept until they were used, under constant temperature conditions $\left(31^{\circ} \mathrm{C}\right)$ and on an 8:16 light-dark cycle similar to the rearing conditions used by Heitler et al. (1977). Only adults having reached sexual maturity were used in experiments; animals that had undergone their final ecdysis more than a month previously generally gave more stable intracellular recordings.

\section{Physiology}

Preparations. Preparations were mounted dorsal side up with insect pins on a cork substrate. Hindlegs were secured against the cork with Plasticine in a position that left the femoral-tibial joint free to flex and extend fully after removal of the distal tibia. The thorax and anterior abdomen were slit longitudinally along the dorsal midline, and the ventral nerve cord and thoracic ganglia were exposed by spreading the nota and flight muscles laterally and removing the gut and overlying tissues and cuticular apodemes. The meso- and metathoracic ganglia were supported on a wax-covered stainless steel platform. A single silver hook electrode around each pro-mesothoracic connective enabled the recording of spikes in the large axons of 2 interneurons, the descending contralateral movement detector (DCMD) and the G neuron, as well as the stimulation of descending axons (see "Results, Voltage threshold"). A pair of copper wires, enamel coated except at their tips, was inserted into each metathoracic tibia; these permitted the stimulation of the axon of the FETi motoneuron and the recording of electromyogram (EMG) activity in the flexor and extensor muscles of the tibiae.

In some of the experiments on changes in FETi's threshold with heating, preparations were mounted ventral side up and recordings were made from the soma of FETi located on the ventral surface of the ganglion (Burrows and Hoyle, 1973). The ventral approach to the ganglion involved far less extensive dissection than did the dorsal approach used for neuropil recordings. In particular, it was possible to record from the soma of FETi with minimal disturbance of the tracheae supplying air to the ganglion. In some preparations, the large trachea that enters the ganglion close to FETi was pulsating visibly with each ventilatory contraction of the thorax and abdomen. Thus, with ventral preparations, we could be reasonably certain that the ganglion was afforded an adequate oxygen supply; in contrast, with dorsal preparations, it was difficult to assess the extent to which respiratory gas exchange occurs. 
Saline and temperature control. Preparations were perfused continuously with an orthopteran saline: 147 mM NaCl, $10 \mathrm{~mm} \mathrm{KCl}, 4 \mathrm{~mm} \mathrm{CaCl}_{2}, 3 \mathrm{~mm} \mathrm{NaOH}$, and 10 mu HEPES, pH 7.2. (In early experiments, a similar saline, buffered with $4 \mathrm{~mm} \mathrm{NaHCO}$ and $6 \mathrm{~mm} \mathrm{NaH} \mathrm{NaO}_{4}$ instead of the $\mathrm{NaOH}$ and HEPES, was used; however, because the carbonate and phosphate formed a precipitatc with $\mathrm{Ca}^{2+}$ and the amount of precipitation increased with heating, the saline buffered with HEPES was preferable.) Temperature was controlled by using a mixture of salines from warm $\left(40^{\circ} \mathrm{C}\right)$ and cool $\left(4^{\circ} \mathrm{C}\right)$ reservoirs in different proportions and was monitored with a thermocouple or thermistor in the hemocoel. Body temperatures were varied over a range from $8^{\circ}$ to $35^{\circ} \mathrm{C}$. Temperature shifts over a given range were carried out in both the cooling and warming directions; once a temperature shift had been completed (which usually required 30 to 120 sec), the temperature effects on the properties that we studied were independent of the direction of the change. Generally, 3 min were allowed after a temperature change before making measurements.

Intracellular recordings. Intracellular recordings were made by penetrating neurons in their larger neuropilar processes using $1 \mathrm{~m}$ KAc-filled electrodes with DC resistances of 40 to 80 megohms. Identification of leg motoneurons was made by correlating movements of the tibia with spikes in the cell from which we were recording. For instance, each spike in FETi produced a rapid extension movement of the hind tibia. Flexor motoneurons were, in addition, identified by correlating the intracellularly recorded spikes in the motoneuron with excitatory junction potentials (EJPs) recorded with the EMG electrodes in the femur. The $\mathrm{G}$ neuron was identified by correlating its intracellularly recorded spikes produced in response to auditory stimuli with its large axon spikes visible in extracellular recordings from the pro-mesothoracic connective (see Fig. 8); in such connective recordings, the spikes in the $G$ neuron generally appeared about one-half to one-third the size of the spikes in the DCMD. The $\mathrm{C}$ neuron was identified by its sensory inputs (Pearson and Robertson, 1981) and by the close proximity of the midline site at which it was penetrated to the large medial neurite of the $G$ neuron; this proximity was evident because, prior to penetrating the $\mathrm{C}$ neuron, the microelectrode recorded an extracellular field potential associated with each $G$ neuron spike in the ventral nerve cord. Furthermore, in some experiments, the $\mathrm{C}$ neuron was identified more reliably by correlating its intracellular spikes with its large extracellular axon spikes recorded with an extracellular electrode against one mesometathoracic connective. We identified the $M$ neuron as described by Pearson et al. (1980).

To record from auditory receptors, we penetrated their axons in the auditory nerve, nerve 6 , approximately 0.5 $\mathrm{mm}$ posterior to where it enters the metathoracic ganglion. Although, due to the small size of the receptor axons, these "partial" intracellular penetrations yielded only small spike amplitudes, they were adequate for reliable recording of spike responses from single receptors.

Voltage and current threshold measurements. Measurements of voltage and current thresholds were made using stimuli spaced at 3- to 5-sec intervals. The spike activity in experiments on threshold and repetitive firing was determined using one or more of the following: (1) intracellularly recorded spikes, (2) extracellularly recorded spikes from the connective in the case of the $G$ neuron, (3) EJPs recorded with EMG electrodes in the case of leg motoneurons, and (4) rapid extension movements of the tibia in the case of FETi. In experiments on FETi, threshold was not measured when the tibia was flexed beyond about $50^{\circ}$ relative to the femur since, during extreme flexion, FETi's threshold is reduced (Heitler et al., 1977) and, in addition, the spikes in FETi may fail to produce movement of the fully flexed tibia.

Input resistance. Input resistance was measured in a small number of experiments using single microclectrodes and a DAGAN 8100 amplifier. Resistance determinations were made both with the standard balanced bridge technique and with 1 to $3 \mathrm{kHz}$ switching between current passing and voltage measuring. With either method, it was critical to use a driven shield (silver paint coating) around electrodes to reduce the electrode time constant.

Repetitive firing patterns. Patterns of repetitive firing in response to injected currents were analyzed directly from photographic records. In the case of $1 \mathrm{G}$ neuron, a computer analysis of its spike trains was carried out with a PDP 11 computer.

$G$ neuron responsiveness. The responsiveness of the G neuron to auditory stimuli was measured by presenting 200-msec pulses of broad band noise at 15-sec intervals. Signals were generated by a random noise generator (General Radio Co. model 1390-B), amplified by a Sanken SI-1010G amplifier, and transduced by a tweeter (Speakerlab model D'T-101). Stimulus intensity was 65 $\mathrm{db}$ and covered a range from 2 to $23 \mathrm{kHz}$ (intensity was measured with a Bruel and Kjaer sound level meter, model 2209, equipped with an octave filter set, type 1613). Similar experiments on the sensitivity of auditory receptors used a Motorola piezo ceramic speaker as a transducer and 100-msec broad band (2.5- to $40-\mathrm{kHZ}$ ) noise stimuli. Spikes in the G neuron were recorded extracellularly from the pro-mesothoracic connective, discriminated electronically with a window circuit, and slored as dots in a raster display.

\section{Results}

\section{Spike threshold}

One possible explanation for the increase in the activity of central neurons with warming would be a reduction in their voltage or current threshold for initiating an action potential. If less depolarization or less excitatory synaptic current were required to fire an action potential in a neuron when it is warm than when it is cool, the cell would tend to be more active when warm. We tested this by measuring the threshold of neurons in the meso- and metathoracic ganglia while varying temperature. The 2 cells most extensively studied in these experiments were the fast extensor tibiae (FETi) motoncuron to the metathoracic leg (Burrows and Hoyle, 1973) and the G neuron, a mesothoracic auditory interneuron.

Voltage threshold. To determine the voltage threshold 
of a neuron with a single intracellular electrode, we applied brief current stimuli to the pro-mesothoracic connectives. Such stimuli resulted in compound postsynaptic potentials (PSPs) in the neuron from which we were recording. The current strength was adjusted until it excited enough presynaptic axons in the connectives to produce a just threshold PSP. Voltage threshold was defined as the average of the amplitudes of the smallest PSP producing a spike and the largest PSP failing to fire an action potential. Thus, threshold is relative to resting potential. Examples of records from such experiments done at two different temperatures are shown in Figure 1 for an FETi neuron, a $G$ neuron, and an $M$ neuron. An increase in temperature was not accompanied by a decrease in the voltage threshold (at least within the temperature range studied: $15^{\circ}$ to $34^{\circ} \mathrm{C}$ ). Rather, a small rise in the voltage threshold usually was seen (Figs. 1 and 2 ).
For the 6 FETi neurons and the $3 \mathrm{G}$ neurons shown in Figure 2, the voltage threshold rose an average of $1.1 \mathrm{mV}$ $(\mathrm{SD}=0.83)$ and $1.3 \mathrm{mV}(\mathrm{SD}=0.33)$, respectively, per $10^{\circ} \mathrm{C}$. We cannot distinguish whether this increase in the voltage threshold with heating was due to a change in the absolute threshold potential or to a hyperpolarization of the resting potential since our recordings of DC potential were not sufficiently stable to allow accurate detection of changes as small as 1 to $2 \mathrm{mV}$; we could determine only that, with $10^{\circ} \mathrm{C}$ shifts in temperature, there were no consistent changes in the resting potential larger than 2 to $3 \mathrm{mV}$.

Current thresholds. Current thresholds were measured by injecting current pulses through the intracellular electrode and increasing the intensity until it was just sufficient to produce a spike during some of the pulses. In both the FETi motoneuron and the G interneuron, the
A
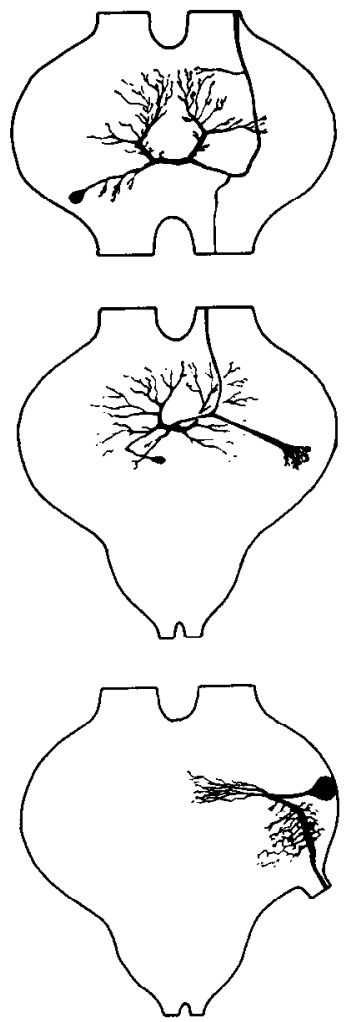

B

$G$ neuron $20^{\circ} \mathrm{C}$
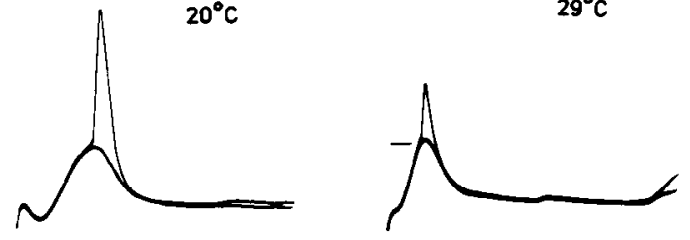

$M$ neuron

$23^{\circ} \mathrm{C}$

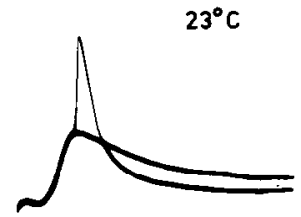

FETi

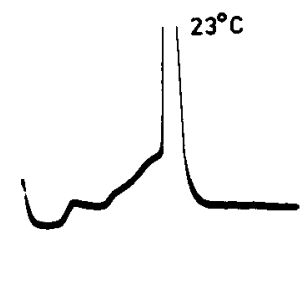

$29^{\circ} \mathrm{C}$

$32^{\circ} \mathrm{C}$
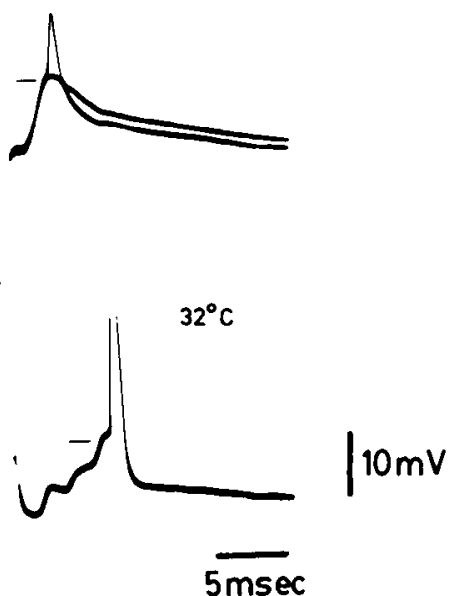

Figure 1. The effect of temperature on the voltage threshold of central neurons. $A$, The anatomy of 3 of the neurons studied; each cell is shown in the ganglion in which it originates. From top to bottom: the G neuron, an auditory interneuron in the second, or mesothoracic, ganglion; the $\mathrm{M}$ neuron, a multimodal interneuron that may trigger the rapid extension of the hind tibia during the jump in the third, or metathoracic, ganglion; and the fast extensor tibiae (FETi) motoneuron of the hindleg in the metathoracic ganglion. $B$, Compound excitatory postsynaptic potentials (EPSPs), which bring each of these 3 neurons to threshold, allow measurements of their voltage threshold relative to their resting potential at different temperatures. The pairs of superimposed traces from experiments on the $\mathrm{G}$ and $\mathrm{M}$ neurons contain 2 consecutive PSPs, only 1 of which resulted in an action potential. The bars next to the records from the higher temperatures indicate the voltage thresholds at the lower temperatures as determined from the records on the left. Note the small increase in the voltage threshold with heating in these 3 neurons. The striking reduction in the amplitude of the action potentials recorded from the $G$ and $M$ neurons at the higher temperatures results from the electrodes penetrating these cells in their inactive regions; the passive conduction of the spike to our recording site is impaired at higher temperatures probably due to both the reduced input resistance and the reduced spike duration. At different temperatures, different strength current stimuli were required to elicit the threshold EPSPs; furthermore, these EPSPs may have heen produced by a different number of presynaptic axons at each temperature. (The Iucifer fills on which the drawings in $A$ are based were done in animals different from those used in the experiments in $B$.) 
A

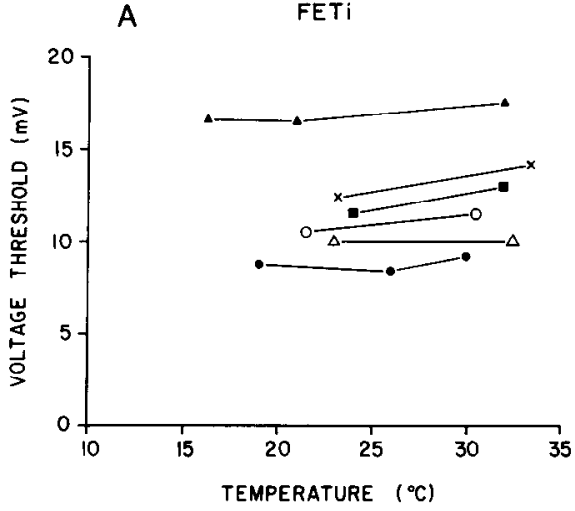

B

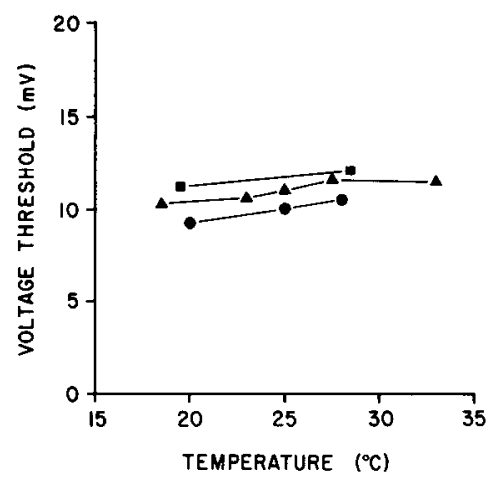

Figure 2. Voltage threshold versus temperature. The voltage threshold relative to the resting potential is plotted for 6 FETi neurons in $A$ and for $3 \mathrm{G}$ neurons in $B$. Note that the voltage threshold increased slightly in all of these cells except for 1 FETi neuron which showed no change.

A

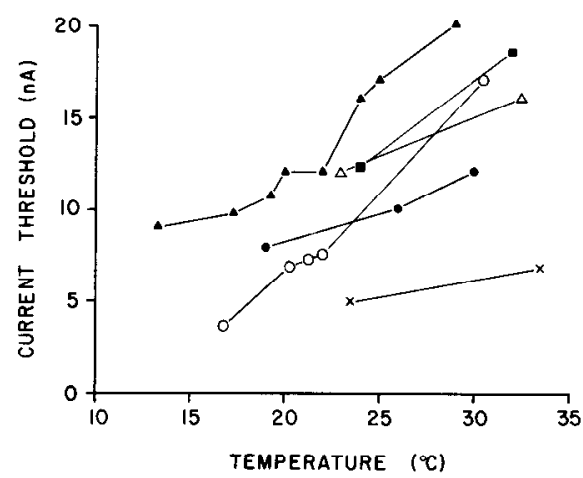

B $\quad G$ neuron

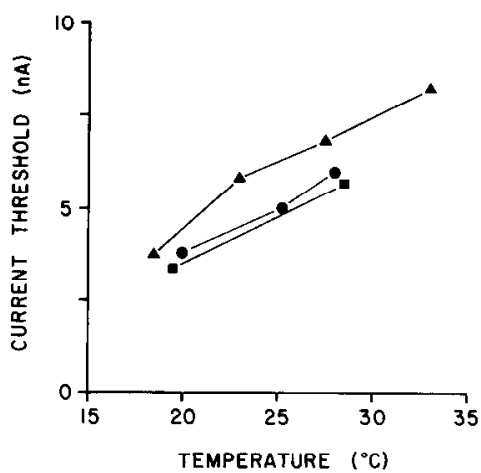

Figure 3. Current threshold versus temperature. FETi neurons and G neurons show proportionally similar increases in the current threshold with increasing temperature. (Note the difference in the current scales.) The pulse durations used for the threshold measurements were $100 \mathrm{msec}$ for the FETi neurons and $10 \mathrm{msec}$ for the G neurons. These data are from the same 9 cells as in Figure 2 (the cells are identified with corresponding symbols).

current thresholds increased markedly with heating (Fig. 3). For example, in 8 FETi neurons, the current threshold rose by an average of $6.8 \mathrm{nA}$ per $10^{\circ} \mathrm{C}(\mathrm{SD}=3.0)$. Although the absolute increase in the threshold of the $\mathrm{G}$ neurons was much smaller-a mean change of $2.9 \mathrm{nA}$ per $10^{\circ} \mathrm{C}(\mathrm{SD}=0.45)$ for the 3 cells in Figure $3 B$-the relative increases in both neuron types were quite similar. Current thresholds for FETi and the G neuron increased on the average by a factor of 1.6 and 1.8, respectively, for similar $10^{\circ} \mathrm{C}$ rises in temperature (over a range from $19^{\circ}$ to $34^{\circ} \mathrm{C}$ ). The increases in the current threshold with heating occurred independent of the current pulse duration used (Fig. 4).

Increases in the current threshold with rising temperature were observed in a total of 13 experiments on FETi motoneurons (7 in Locusta migratoria and 6 in Schistocerca gregaria, all done with recordings from neuropilar processes) and in 7 experiments on G neurons. A substantial increase in the current threshold with warming was a widespread phenomenon, occurring in a number of other central neurons, including flight motoneurons, metathoracic flexor tibiac motoneurons, and the $\mathrm{C}$ neuron (see below). On the other hand, one identified metathoracic interneuron, the $M$ neuron, behaved differently in some animals. In 5 of a total of 8 experiments, the

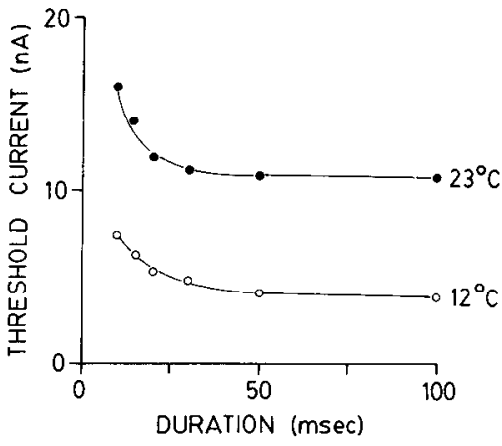

Figure 4. Strength-duration curves for an FETi neuron at two different temperatures. Note that the current threshold increased with heating independent of the stimulus duration used.

current threshold changed little as the temperature was raised. In the other 3 experiments, the $M$ neuron showed a rise in the current threshold with increasing temperature roughly comparable to that seen in the cells described above.

Input resistance. Input resistance was measured in a few experiments with single electrodes in the neuropilar processes of motoneurons. With heating, there were re- 
ductions in input resistance accompanying the increases in the current threshold. For example, in 1 flight motoneuron, the input resistance changed from about 1.8 megohms at $16^{\circ} \mathrm{C}$ to about 1.0 megohm at $28^{\circ} \mathrm{C}$, while the current threshold rose from 4.7 to $7.8 \mathrm{nA}$. Thus, changes in resting membrane resistance may account at least for a substantial part of the changes in the current threshold described above.

Soma experiments. We were surprised by our finding that the current threshold of central neurons increased with heating. That warm neurons should require more injected current to initiate an action potential was unexpected, given our behavioral observations of increased locomotory activity in our grasshoppers as the ambient temperature was raised about $22^{\circ} \mathrm{C}$. Furthermore, our results disagreed with those of Heitler et al. (1977) who found that, in Schistocerca nitens, both the current and voltage thresholds of FETi dropped as the temperature increased. The data of Heitler et al. (1977) were obtained with microelectrodes in the somata of FETi neurons, while our measurements had been made with electrodes in the main neuropilar processes of the cells.

We repeated our experiments on changes in FETi's current threshold with temperature, mounting animals ventral side up and penetrating FETi in its soma. In an effort to replicate the study of Heitler et al. (1977) more closely, the soma experiments were done on $S$. nitens as well as on Schistocerca americana. Determinations of the current threshold in the soma of FETi were made in a total of 10 animals. In only 1 animal, an $S$. nitens individual, did we see a decrease in FETi's current threshold with heating. In a 2nd animal, FETi showed little change in threshold with temperature. The remaining 8 FETi neurons behaved similarly to the cells studied with neuropil recordings (i.e., their current thresholds increased as the temperature was raised). Thus, in our experiments on thoracic interneurons and motoneurons, with the exception of a single FETi neuron, we have found no evidence for a reduction in spike threshold that could account for the increased activity in the CNS of warm grasshoppers.

\section{Frequency-current relation}

One alternative explanation for the increased spike activity in warm central neurons is that there is a temperature-dependent shift in the processes controlling repetitive firing, causing neurons to fire faster at higher temperatures once they are depolarized above threshold. We describe here the effect of temperature on the firing patterns of neurons stimulated with suprathreshold current pulses.

Figure 5 consists of records from an experiment on a metathoracic intermediate flexor tibiae motoneuron that was stimulated with current pulses over a range of intensities. The frequency-current (f-I) relations for this neuron at two temperatures have been plotted in Figure 6. During each 100 -msec current pulse, this flexor motoneuron's firing rate adapted substantially. In describing the responses of this neuron and others that adapt, we consider separately their initial peak spike frequency and their final, steady state, spike frequency at the end of the current pulse. When stimulated with low, just supra-

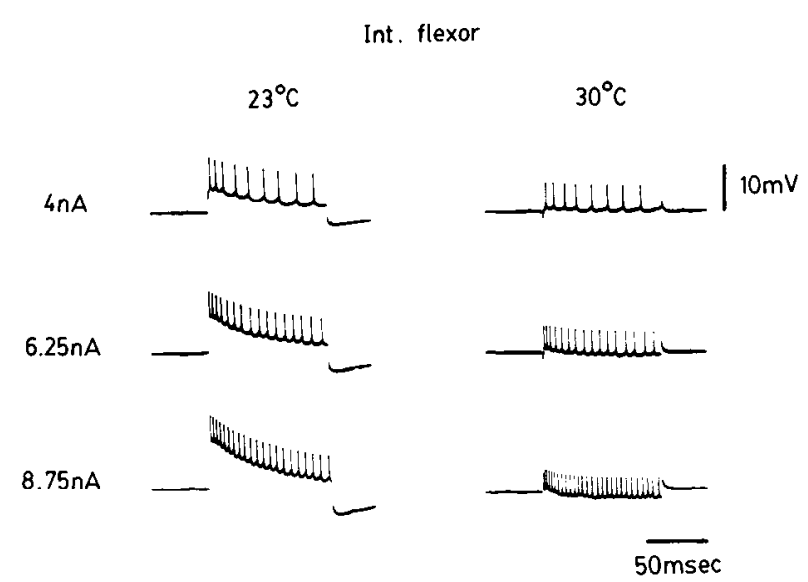

Figure 5. Effect of temperature on repetitive firing in a flexor motoneuron. Each trace shows the intracellularly recorded spikes in this metathoracic intermediate flexor motoneuron produced in response to the injection of a 100-msec depolarizing current pulse. Note that, with low stimulus current strengths, heating reduced this cell's firing frequency, while, with higher current strengths, heating increased its spike frequency. Also note the spike frequency adaptation during each $100-\mathrm{msec}$ current pulse. (The temperature effect on spike size is a consequence of our recording from an inactive region of the neuron as in Fig. 1. The drift in base line during current pulses is due to $\mathrm{AC}$ coupling of oscilloscope.)

threshold currents, this flexor neuron had a higher initial spike frequency at $23^{\circ}$ than at $30^{\circ} \mathrm{C}$ (Figs. 5 and $6 \mathrm{~A}$ ). However, with increasing current strength, the initial spike frequency rose more steeply at the warmer temperature until, with currents above about $5 \mathrm{nA}$, the cell had a higher peak frequency when warm (Figs. 5 and 6A).

In comparison with the peak spike frequency, the steady state firing rate changed little with temperature. With weak current pulses, this flexor neuron's adapted spike frequency was only slightly higher at $23^{\circ} \mathrm{C}$ (Figs. 5 and $6 B$ ). Although the steady state $\mathrm{f}-\mathrm{I}$ curves for the two temperatures cross one another, only with the largest current strengths used was the adapted frequency substantially higher at $30^{\circ} \mathrm{C}$ (Figs. 5 and $6 B$ ). One way to characterize the effect of temperature on the f-I relation is to calculate the change in the slope of the curves (in their linear regions) with warming. The slope of the f-I relation for the peak frequency of this flexor increased by a factor of 2.2 as the temperature was raised from $23^{\circ}$ to $30^{\circ} \mathrm{C}$ (Fig. $6 \mathrm{~A}$ ). The slope of the steady state $\mathrm{f}$-I curves changed relatively less with warming, increasing by a factor of 1.3 for this $7^{\circ} \mathrm{C}$ rise in temperature.

We have seen a similar steepening of both the peak and the steady state f-I relations with increasing temperature in most of the other central neurons that we have studied; these include intermediate and fast flexor tibiae motoneurons, the FETi motoneuron, and 2 interneurons-the $M$ neuron and an unidentified interneuron, studied in a single experiment, that was located in the mesothoracic ganglion and was sensitive to light dimming. The f-I curves from $1 \mathrm{M}$ neuron in which threshold changed little with warming are presented in Figure 7. Since this cell's f-I curves for the two temperatures intersected near threshold, its firing frequency was higher 


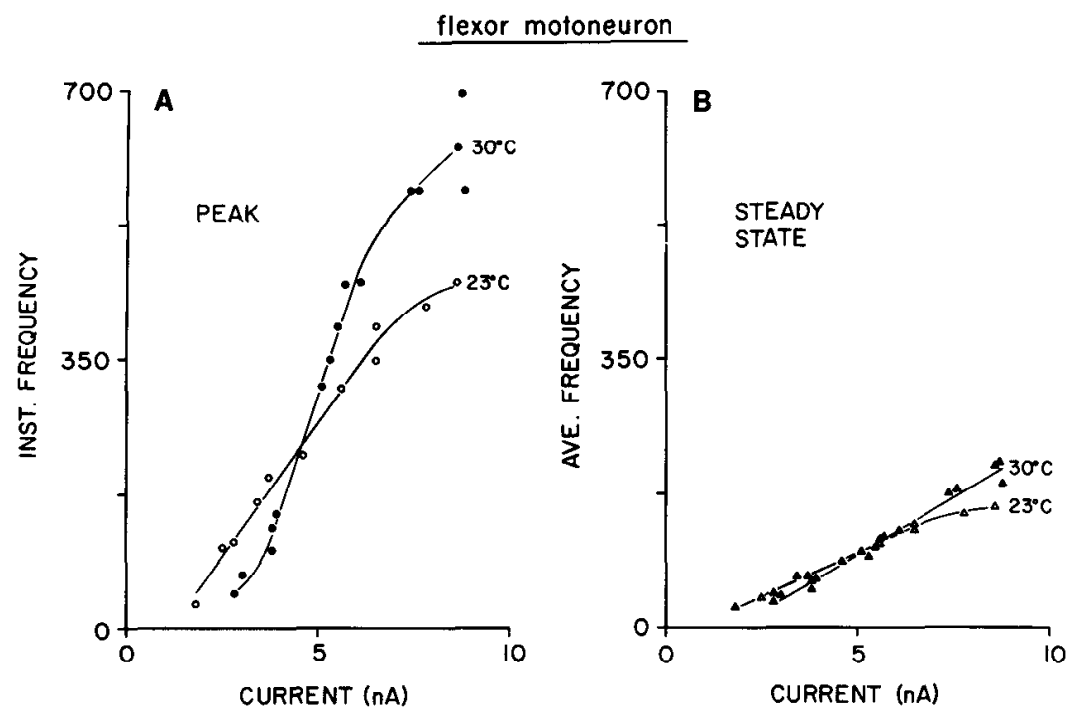

Figure 6. Effect of temperature on the $\mathrm{f}$-I relation of a flexor motoneuron. $A$, Instantaneous peak firing frequency, the reciprocal of the shortest interspike interval occurring at the start of the spike train during a 100-msec depolarizing current pulse, versus current strength. $B$, Steady state firing frequency, the reciprocal of the mean of the last several interspike intervals prior to the termination of the current pulse, versus current strength. In these f-I curves and those in subsequent figures, the lowest currents for which points are plotted are slightly above threshold at each temperature. Each point in this figure and in Figures 7 and 9 represents the spike frequency during the response to a single current pulse. Linear portions of the curves were fitted by the least squares method; the remaining portions were fitted by eye. Note (1) that heating increases the slopes of both the peak and steady state f-I curves; (2) that there is relatively less change with temperature in the slope of the steady state f-I relation; in fact, this neuron's steady state firing frequency is quite well temperature compensated; and (3) that, when stimulated with current strengths below $4 \mathrm{nA}$, this cell fired faster at $23^{\circ}$ than at $30^{\circ} \mathrm{C}$. These data are from the same neuron as those in Figure 5.

\section{M neuron}
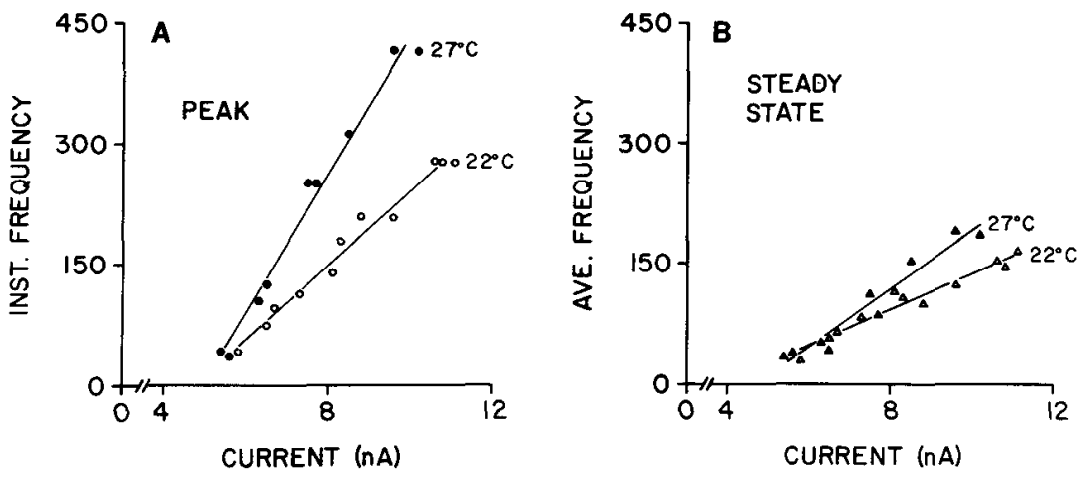

Figure 7. Effect of temperature on the f-I relation of the $\mathrm{M}$ neuron. In this $\mathrm{M}$ neuron, heating had little effect on either its threshold or its low frequency repetitive firing. Note the steepening of both the peak and steady state f-I curves at $27^{\circ} \mathrm{C}$. (See the legend to Fig. 6 for an explanation.)

at $27^{\circ}$ than at $22^{\circ} \mathrm{C}$ for all suprathreshold current strengths.

Temperature effects on the steady state firing of the $C$ and $G$ neurons. Two mesothoracic interneurons, the $G$ neuron and the $\mathrm{C}$ neuron, differed from the other cells studied in the way in which their steady state f-I relations were affected by temperature. Like the neurons described above, when stimulated with sufficiently strong current pulses, the $G$ neuron had a faster initial firing rate at higher temperatures (Figs. 8 and 9A). However, independent of current intensity, its steady state firing frequency was always lower at the higher temperatures (Figs. 8 and $9 B$ ). As seen in Figure $9 B$, the $G$ neuron usually showed a slight decrease in the slope of its steady state $f-I$ curve with warming.

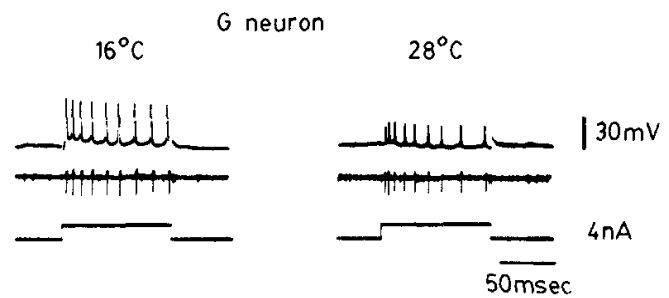

Figure 8. Repetitive firing in a $\mathrm{G}$ neuron at two temperatures in response to depolarizing current pulses. Upper trace, Intracellular recording from a neuropilar process; middle trace, extracellular recording from the pro-mesothoracic connective; lower trace, current record (4-nA pulses). Note that, while the initial, peak frequency is higher at $28^{\circ} \mathrm{C}$, the adapted, steady state frequency is higher at $16^{\circ} \mathrm{C}$. 


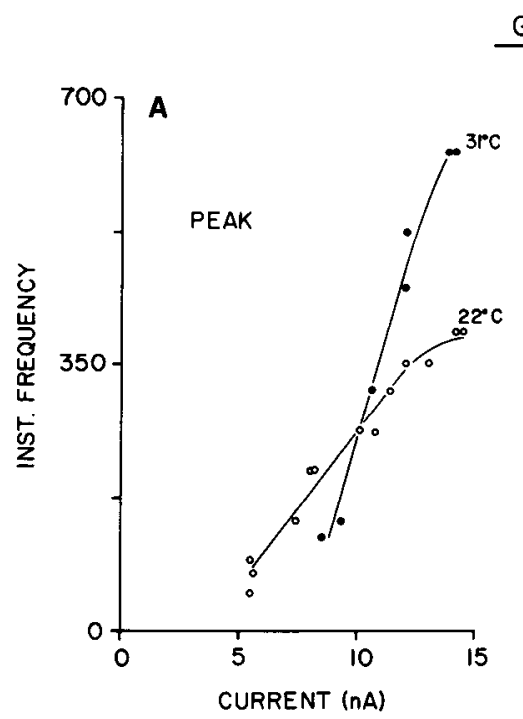

G neuron

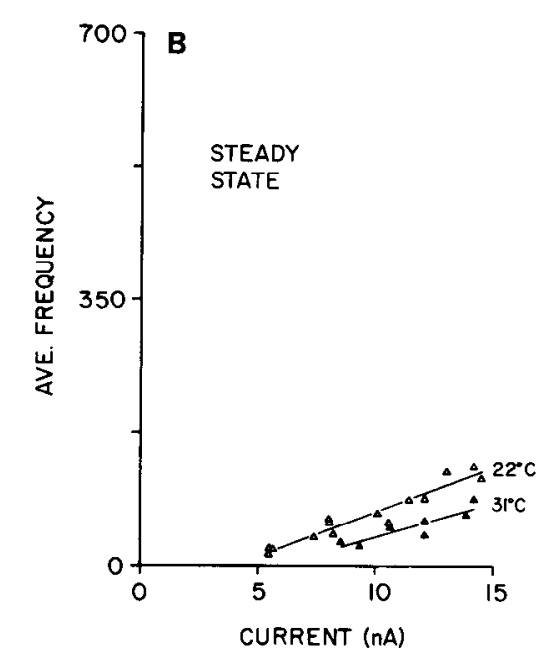

Figure 9. Effect of temperature on the f-I relation of the G neuron. Note that, in contrast to the steepening of the peak f-I curve which accompanies heating, there is no increase in the slope of the steady state f-I curve. (See the legend to Fig. 6 for an explanation.)

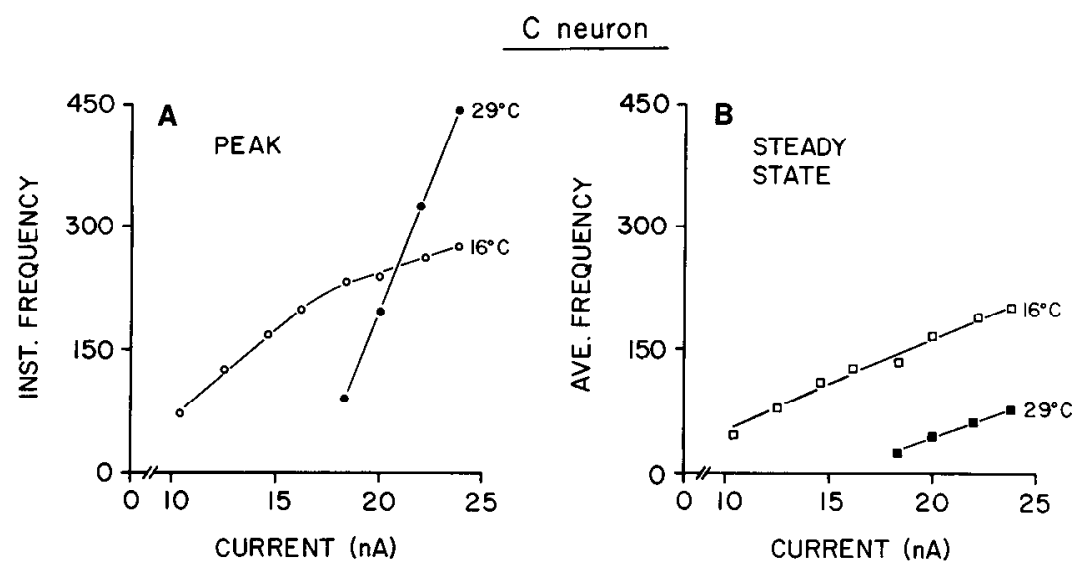

Figure 10. Effect of temperature on the $\mathrm{f}$-I relation of the $\mathrm{C}$ neuron. Note the difference in the effect of temperature on peak and steady state firing. Each point represents the mean of 5 to 19 responses to a single current strength. (See the legend to Fig. 6 for an additional explanation.)

The $\mathrm{C}$ neuron behaved similarly to the G neuron. As in the $\mathrm{G}$ neuron, the slope of the $\mathrm{C}$ neuron's peak $\mathrm{f}-\mathrm{I}$ curve increased with warming (Fig. 10A). In both cells, the steady state f-I curves were shifted down with an increase in temperature (Figs. $9 B$ and $10 B$ ); since there was no steepening of the slopes of their steady state f-I relations with warming, high and low temperature steady state f-I curves did not cross. Thus, in the $\mathrm{C}$ and G neurons, with a rise in temperature, there is a sufficient increase in adaptation to more than offset the temperature-dependent increase in the initial spike frequency.

To better understand the increase in adaptation with temperature, we performed a more detailed analysis of the spike trains produced by a single $G$ neuron at $16^{\circ}$ and at $28^{\circ} \mathrm{C}$. At the higher temperature, there was both a decrease in the time constant for adaptation and an increase in the extent of adaptation. It is possible that the greater adaptation seen at $28^{\circ} \mathrm{C}$ simply might have been due to the $\mathrm{G}$ neuron's having a higher initial firing rate when warm (at least for most suprathreshold current strengths); that is, a faster initial spike frequency might, independent of temperature, have resulted in a decreased final spike frequency. However, even when the initial frequencies were comparable at the two temperatures, both the rate and extent of adaptation were increased at $28^{\circ} \mathrm{C}$ as compared with those at $16^{\circ} \mathrm{C}$ (Fig. 11). In summary, when the $\mathrm{G}$ and $\mathrm{C}$ neurons were stimulated with sufficient current, a rise in temperature resulted in both a substantial increase in the initial firing rate and a large, opposing increase in the rate and amount of adaptation.

\section{Synaptic potentials}

In addition to changes in the intrinsic firing properties of single neurons with increasing temperature, there also could be changes in synaptic transmission which could contribute to the increased activity of central neurons in warm animals. We have looked at the effect of temperature on excitatory synaptic connections between the descending contralateral movement detector (DCMD) neuron, a descending protocerebral visual interneuron, and 3 identified postsynaptic neurons in the thorax. In each of these postsynaptic cells, the $M$ neuron, the FETi 
neuron, and the $\mathrm{C}$ neuron, with heating, we consistently saw a decrease in the duration of the excitatory postsynaptic potential (EPSP) from the DCMD. For example, in 1 experiment on a $\mathrm{C}$ neuron, the results of which are presented in Figure 12, the duration of the EPSP at $50 \%$ maximum amplitude decreased by a factor of 1.7 when the temperature was raised from $18^{\circ}$ to $29^{\circ} \mathrm{C}$. There was

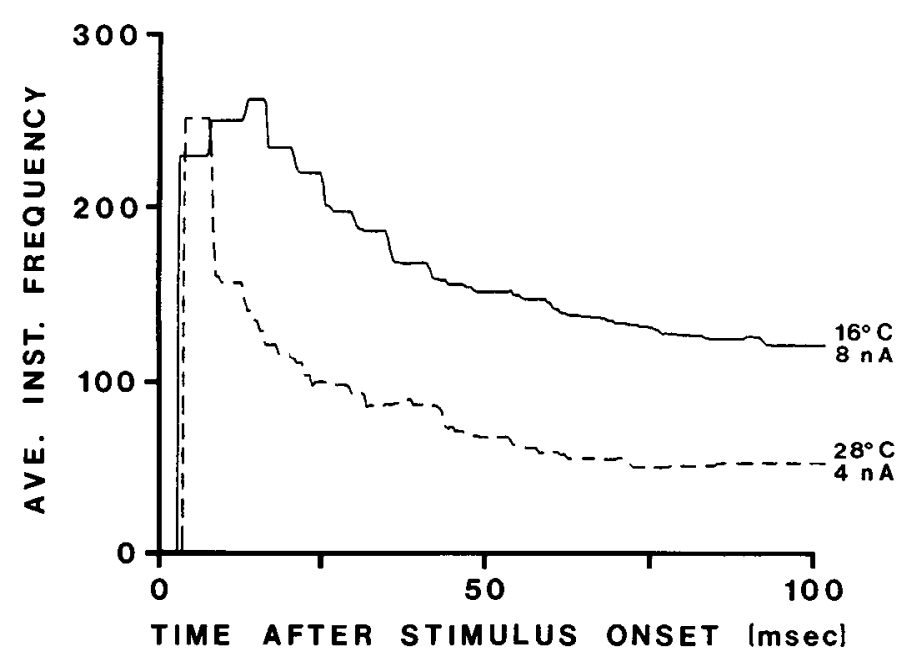

Figure 11. Effect of temperature on spike frequency adaptation in the $G$ neuron. Instantaneous spike frequency is plotted as a function of time from the start of the depolarizing current pulse for two current strengths that produced similar initial spike frequencies at these two temperatures: $8 \mathrm{nA}$ at $16^{\circ} \mathrm{C}$ (solid line) and $4 \mathrm{nA}$ at $28^{\circ} \mathrm{C}$ (broken line). Note the high initial rate of adaptation at $28^{\circ} \mathrm{C}$ and the difference between the final adapted frequencies at the two temperatures. The data are averaged from 10 spike trains in response to identical current stimuli; average instantaneous frequency is the reciprocal of the mean interspike interval for the 10 spike trains calculated for each 0.4-msec time bin.

A

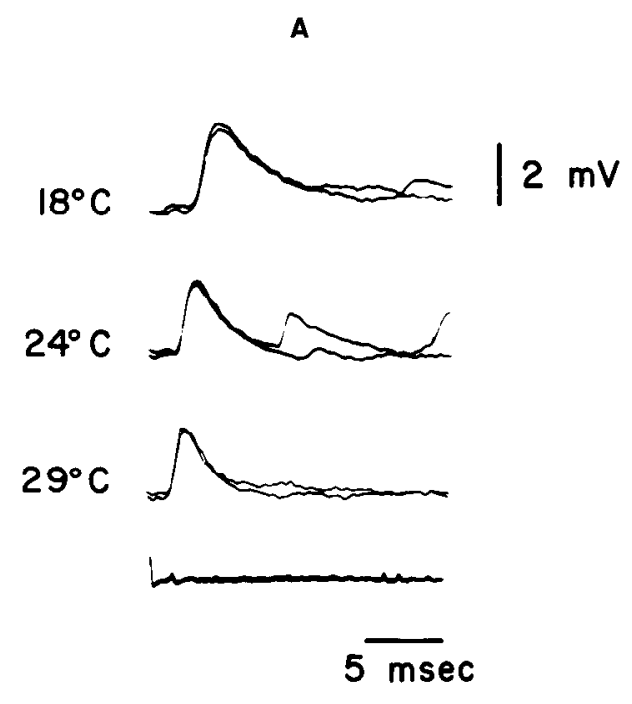

also a tendency for the size of the EPSP to decrease with warming, although the effect of temperature on EPSP amplitude was both smaller and less consistent than on EPSP duration. In this C neuron, the EPSP amplitude decreased by a factor of 1.3 for this same $10^{\circ} \mathrm{C}$ rise in temperature. In experiments on FETi neurons and $M$ neurons, in some instances, there were small reductions in EPSP amplitude with increasing temperature; in other cases, no measurable change in size was observed. In general, no increase in EPSP amplitude occurred that could cause spikes in the DCMD to be more effective in exciting postsynaptic neurons when grasshoppers are warm. In addition, the reduced EPSP duration at higher temperatures would decrease the probability of summation of temporally distributed synaptic inputs.

\section{Effect of temperature on the auditory response of the $G$ neuron}

Early in this study, we determined the response of the $G$ neuron to auditory stimuli as a function of temperature. We had hoped to be able to account for any observed changes in the $\mathrm{G}$ neuron's responsiveness in terms of temperature-dependent changes in the neuron's intrinsic firing properties. We presented $200-\mathrm{msec}$ pulses of broad band noise to grasshoppers and recorded the resultant trains of $\mathrm{G}$ neuron spikes with an extracellular hook electrode around the pro-mesothoracic connective. These extracellularly recorded spikes are relatively large and readily distinguishable due to the large diameter of the $\mathrm{G}$ neuron's ascending axon (approximately $12 \mu \mathrm{m}$ ). (See Fig. 8 for examples of spikes recorded from the ventral nerve cord.)

The G neuron's response to broad band noise stimuli was measured at temperatures ranging from $9^{\circ}$ to $32^{\circ} \mathrm{C}$. With increasing temperature, there was an increase in the total number of spikes produced by the $G$ neuron

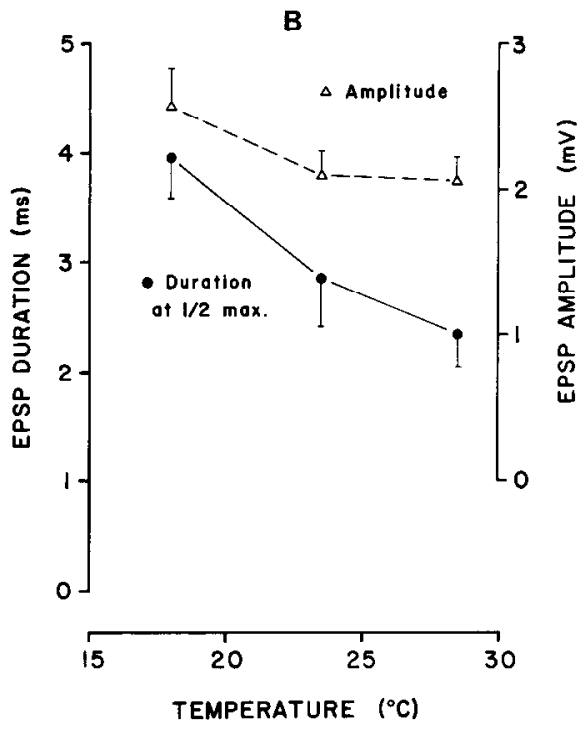

Figure 12. Effect of temperature on EPSPs. A, EPSPs following spikes in the DCMD neuron recorded in the C neuron at three temperatures. Sweeps were triggered on the DCMD spike recorded extracellularly from the pro-mesothoracic connective (lowest trace); at each temperature, two consecutive EPSPs are shown in superimposed traces. $B$, EPSP duration at half-maximal amplitude $(O)$ and EPSP amplitude $(\triangle)$ versus temperature. The data points are the means of 15 to 29 PSPs ( \pm SD). Means were calculated only for EPSPs occurring during low frequency firing in the DCMD since EPSPs are reduced in amplitude during moderate to fast DCMD firing. 
during each stimulus (Fig. 13A). Most of this increase in total spike number was due to a rise with warming in the peak spike frequency at the beginning of the response (Fig. 13B). The G neuron's adapted firing rate, $100 \mathrm{msec}$ after the start of the response, also increased with warming although less steeply than the initial frequency. At temperatures above $20^{\circ} \mathrm{C}$, the adapted firing rate was fairly well temperature compensated; in the neuron for which data are presented in Figure $13 B$, the adapted firing rate was virtually unchanged between $20^{\circ}$ and $30^{\circ} \mathrm{C}$.

The effect of temperature on the adapted firing rate of the $G$ neuron stimulated with sound did not parallel the temperature effect on its steady state firing when it was stimulated directly with depolarizing currents (compare Fig. $9 B$ with Fig. $13 B$ ). As described above, the G neuron's steady state spike frequency during current pulses decreased with increasing temperature. The discrepancy between the effects of temperature on the G neuron's intrinsic steady state firing properties and on its adapted auditory response was due to an increase with heating in the excitatory synaptic input that it received during auditory stimuli.
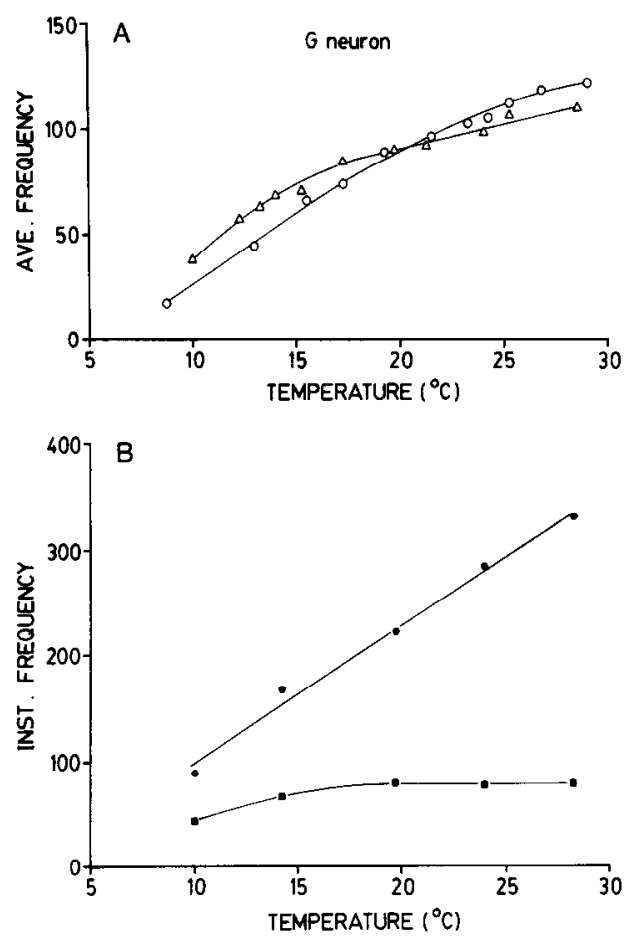

Figure 13. Response of the $\mathrm{G}$ neuron to auditory stimuli as a function of temperature. $A$, Average firing frequency of $\mathrm{G}$ neurons during the presentation of a $200-\mathrm{msec} 65-\mathrm{dB}$ broad band noise stimulus. Each point is the mean of 10 responses; the triangles and circles represent results from two different experiments. Note the increase with heating in the total number of spikes during a stimulus. $B$, Responses of $1 \mathrm{G}$ neuron ( $\triangle$ in $A)$ plotted as initial peak instantaneous frequency (O) and adapted frequency $100 \mathrm{msec}$ after the initiation of the response (D). Each point is the mean of 10 responses. Note that the steady state response of this $G$ neuron to auditory stimuli increased with heating, reaching a plateau at $20^{\circ} \mathrm{C}$; this contrasts with the decline with heating in its steady state frequency during current injection (compare with Fig. $9 B$ ).
Intracellular recordings from a $\mathrm{G}$ neuron during presentations of a constant broad band noise stimulus revealed that it received a larger wave of depolarizing synaptic potentials at $31^{\circ}$ than at $22^{\circ} \mathrm{C}$ (Fig. 14). Since experiments on identified synaptic connections do not indicate an increase in synaptic efficacy at warmer temperatures (Fig. 12), this increase in synaptic input suggests an increased activity in auditory afferents. The $G$ neuron receives at least a substantial portion of its synaptic input from auditory interneurons (Kalmring, 1975; $K$. Pearson, unpublished observations). We therefore cannot distinguish whether the observed increase in synaptic input with heating is due to the effects of temperature on the primary receptors in the ear or on interposed auditory interneurons; for instance, changes in repetitive firing properties are likely to cause first order interneurons receiving strong excitatory input during stimuli to fire at higher frequencies in the warm animal.

However, in another set of experiments in which we recorded from the axons of auditory receptors, we were able to determine directly how the responsiveness of these receptors is affected by temperature. We presented broad band noise stimuli and recorded the spike trains produced by individual receptors at a range of temperatures. Such experiments indicated that increased temperature results in a dramatic enhancement of the responsiveness of the auditory receptors at both threshold and suprathreshold stimulus intensities (Fig. 15). Thus, with heating, there is an increase in the input that central neurons receive from these receptors during auditory stimuli. This increased afferent input must be an important factor in producing the increase in both the initial and the adapted response of the $G$ neuron at higher temperatures. The importance of increased input from these and other sensory receptors in the warm animal is considered further in the discussion.

\section{Discussion}

In grasshoppers, like many insect species, the frequency with which behaviors occur varies greatly with temperature. This study has identified several properties of the central neurons of grasshoppers which show substantial temperature-dependent changes, changes which are likely to contribute to determining how the activities of sets of neurons involved in the control of behavior are affected by temperature. The neuronal properties showing substantial temperature sensitivity include: (1) cur-

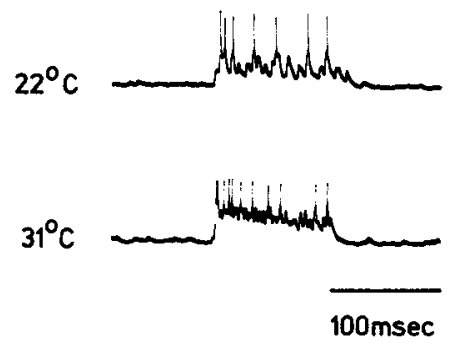

Figure 14. Synaptic input to a G neuron during a broad band noise stimulus at two temperatures. Note that, at the higher temperature, there is an increase in the frequency of synaptic potentials and an increase in the level of depolarization reached by the summed synaptic input during the stimulus. 


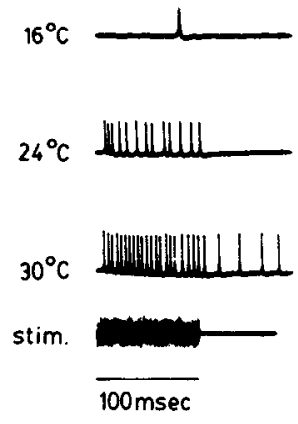

A

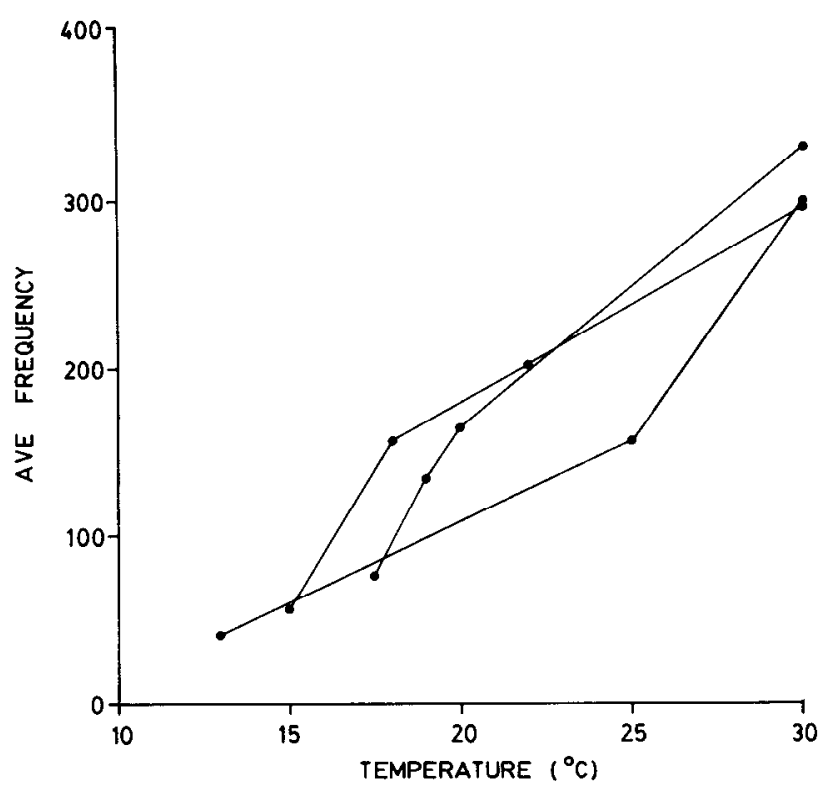

B

Figure 15. Effect of temperature on the sensitivity of auditory receptors to broad band noise stimuli. $A$, Response of an auditory receptor to a $100-\mathrm{msec}$ broad band noise stimulus at three temperatures. Note that this stimulus, which was at threshold for this receptor at $16^{\circ} \mathrm{C}$, elicited quite vigorous responses at warmer temperatures. Further cooling below $16^{\circ} \mathrm{C}$ eliminated the response; thus, the auditory threshold of this receptor was reduced with heating. $B$, Average spike frequency in three auditory receptors during $100-\mathrm{msec} 84-\mathrm{dB}$ broad band noise stimuli at a range of temperatures. Note the dramatic increase in the response of these receptors with heating. (The data in both $A$ and $B$ were obtained from "partial" intracellular penetrations of the receptors' axons in the auditory nerve; see "Materials and Methods, Intracellular recordings").

rent threshold, (2) frequency-current relations for both the initial and the steady state firing rates, and (3) PSP duration. We begin by discussing temperature effects on the spike threshold.

\section{Spike threshold}

One striking effect seen in our experiments was a substantial and linear increase in the current threshold with increasing temperature. The amount that the current threshold rose with heating seems to be proportional to the current strength needed to fire a particular cell type. In the G neurons, the average current needed to produce a spike was considerably less than in the FETi neurons; yet, in both cells, the threshold increased by similar factors with comparable $10^{\circ} \mathrm{C}$ increases in temperature.

Our voltage threshold measurements indicate that there is a small increase in the voltage threshold relative to the resting potential with heating, averaging $1.1 \mathrm{mV}$ per $10^{\circ} \mathrm{C}$ in FETi and $1.3 \mathrm{mV}$ per $10^{\circ} \mathrm{C}$ in the G neuron. There are limitations to our method of determining the voltage threshold. Rather than depolarizing spaceclamped neurons with voltage steps, we relied on excitatory synaptic input to obtain an estimate of the voltage threshold. Both temperature effects on the rate of rise of the compound PSP and temperature-dependent changes in the conduction of the PSP to the spike-initiating zone, due to changes in PSP duration and input resistance, may have complicated our measurement of the voltage threshold as a function of temperature. However, in the case of our recordings from the large neurite of FETi, since our electrodes were electrically close to the spike initiation region, temperature effects on PSP propagation probably had little influence on our estimates of thresh- old. ${ }^{3}$ Therefore, the small increase in the voltage threshold with increasing temperature that we observed in the

\footnotetext{
${ }^{3}$ Two complicating factors may have influenced our results on the voltage threshold. (1) At lower temperatures, the rise time of the compound PSPs was slower (Fig. 1), which would have allowed more time for $\mathrm{Na}^{+}$conductance to inactivate and $\mathrm{K}^{+}$conductance to activate before threshold was reached; thus, given these slower voltage changes, a larger depolarization would have been required to initiate an action potential at the lower temperatures, resulting in an underestimate of the increase in the voltage threshold with increased temperature. (2) Because the cells were not isopotential, the voltage change recorded may well have been attenuated between our recording site and the spike initiation region; this is assuming that the synaptic inputs were situated electrically closer to our electrode than to the point of spike initiation, which is almost certainly the case with the $G$ neuron. (We penetrated the $G$ neuron in its large neurite which crosses the midline connecting the two halves of its dendritic arborization (Fig. 1). To produce a PSP, we stimulated the pro-mesothoracic connective contralateral to the $G$ neuron's axon. Thus, our electrodes were situated between the initial segment of the axon and those dendrites in the subfield ipsilateral to the connective being stimulated.) The attenuation of PSPs would have been greater at the higher temperatures because, with their shorter durations, they would have been less effective in charging the membrane capacitance of the neurite leading to the spike initiation zone; in addition, any decrease in membrane resistance with warming also would have increased PSP attenuation. Thus, at higher temperatures, a larger PSP would have been required to produce a given depolarization at the spike initiation region, resulting in an overestimate of the increase in the voltage threshold with heating. As mentioned in the text, these overestimates of the threshold depolarization at the spike initiation zone are probably small in the case of recordings from the large neurite of FETi where our electrodes were electrically close to the site of spike initiation. In experiments on Schistocerca americana, where the spikes that we recorded often were overshooting, we actually may have been penetrating FETi within the spike initiation region (Gwilliam and Burrows, 1980).
} 
FETi neurons is most probably indicative of actual threshold changes occurring in the region of spike initiation.

We can conclude that, at higher temperatures, a slightly larger amplitude excitatory PSP would have been required to bring one of these central neurons to threshold. Furthermore, since the current threshold decreases with cooling, we would expect a given depolarizing postsynaptic current to be more effective at lower body temperatures.

Our results on the current threshold are consistent with the predictions of Fitzhugh (1966) who investigated the temperature dependence of threshold using a computer model of a space-clamped squid axon. Fitzhugh's (1966) analysis indicated that, for long, rheobasic current stimuli, the threshold should increase with increasing temperature. This prediction of Fitzhugh (1966) was substantiated by experiments of Guttman (1966) on spaceclamped squid axons. Guttman (1966) found that, for rheobasic currents, the threshold increased by a factor of 2.35 per $10^{\circ} \mathrm{C}$ rise in temperature. Since the current stimuli used in our threshold experiments were all relatively long, our results on grasshopper neurons parallel those of Fitzhugh (1966) and Guttman (1966) fairly closely. Despite the similarity between the two systems in the temperature dependence of the spike threshold, there are significant differences between isolated squid giant axons and neurons in intact grasshopper ganglia, differences that are likely to be important in determining how threshold varies with temperature. For instance, there are additional types of ion channels in the somata and neuropilar processes of insect neurons other than those found in squid axons (N. Altekar and T. W. Abrams, unpublished results). Moreover, the excitability of central grasshopper neurons must be influenced by the extracellular environment (e.g., the concentrations of divalent cations), as well as by tonic synaptic inputs, and changes in both of these factors could be expected to accompany temperature shifts.

Input resistance. Of these and other potential factors that may influence the temperature dependence of threshold in grasshopper neurons, the only one that we have examined is input resistance. In the few experiments in which resistance measurements were made, input resistance decreased with heating. Such a reduction in the membrane resistance of central neurons could account for the observed increase in their current threshold in the absence of a parallel changc in their voltage threshold and also could contribute to the observed changes in PSP time course.

Comparison with previous study on Schistocerca nitens. While our results on threshold are not theoretically surprising, it is puzzling that these findings should differ so markedly from those obtained by Heitler et al. (1977) in almost identical experiments on grasshopper neurons. In their grasshoppers, heating initially resulted in an increase in threshold similar to the threshold increase seen in our animals. Then, after approximately $100 \mathrm{sec}$, the threshold began to decrease until it declined below the previous level at the cooler temperature (Heitler et al., 1977, Fig. 10); this second delayed phase of the temperature response was absent in our experiments. Cooling their preparations produced reciprocal effects: an initial threshold decrease, much as in our animals, followed by a subsequent threshold increase. In our experiments, we observed no secondary delayed response to temperature changes. Efforts to reproduce the results of Heitler et al. (1977) by varying experimental parameters were unsuccessful. We consistently saw an increase in FETi's threshold with heating in Locusta migratoria and in two populations of Schistocerca americana. In the experiments that we performed on the species studied by Heitler et al. (1977), S. nitens, the current threshold increased with warming in 2 out of 4 animals; a dccrease in threshold was seen in only 1 S. nitens individual (Fig. $16 A$ ).

Did we fail to see a slowly developing reduction in threshold with heating because our preparations were damaged and behaved abnormally? Had our dissections interfered with the normal physiology of the cells studied, we would expect that this would be a variable effect and that the neurons might deteriorate with time. On the contrary, in more than 42 experiments on various interneurons and motoneurons, including 22 experiments on FETi neurons (Fig. 16A), we saw no reduction in threshold with heating despite the fact that we measured the threshold for a minimum of $4 \mathrm{~min}$ and occasionally for up to $30 \mathrm{~min}$ following a temperature change. In only the single $S$. nitens individual mentioned above did we observe a decrease in the current threshold of a central neuron with heating. Preparations usually survived $4 \mathrm{hr}$ or longer, with normal sensory responses in higher order interneurons. Furthermore, neuropil penetrations were quite stable and yielded repeatable results for as long as $3 \mathrm{hr}$. Those preparations in which we recorded from the soma of FETi required relatively little dissection and minimal disturbance of their respiratory gas exchange (see "Materials and Methods"). The vast majority of these soma experiments gave results consistent with those from neuropil experiments, allowing us to be fairly confident that the increase in the current threshold that we observed in neuropil experiments was not a result of an inadequate oxygen supply. The consistency of the responses that we observed, combined with the good health of our preparations and the stability of our penetrations, suggests that the discrepancy between our results and those of Heitler et al. (1977) was not due to our preparations being damaged. It is also unlikely that the findings of the two studies disagreed because of differences in experimental methods since, after discussions with C. Goodman (Department of Biological Sciences, Stanford University, Stanford, CA), we closely replicated the procedures used by Heitler et al. (1977).

We propose instead that the lack of agreement between the two sets of observations may reflect actual differences between species and within the laboratory populations of $S$. nitens that were studied. Two pieces of evidence support this interpretation. First, within each study, some variability was seen in the response of $S$. nitens neurons to temperature changes. Two animals in the Heitler et al. (1977) experiments were atypical in that the current threshold of their FETi neurons exhibited a temperature dependence resembling that commonly seen in our experiments (i.e., their threshold increased with heating (Fig. 16B)). Conversely, 1 S. nitens individual in our study was unusual in that the current threshold of 
A

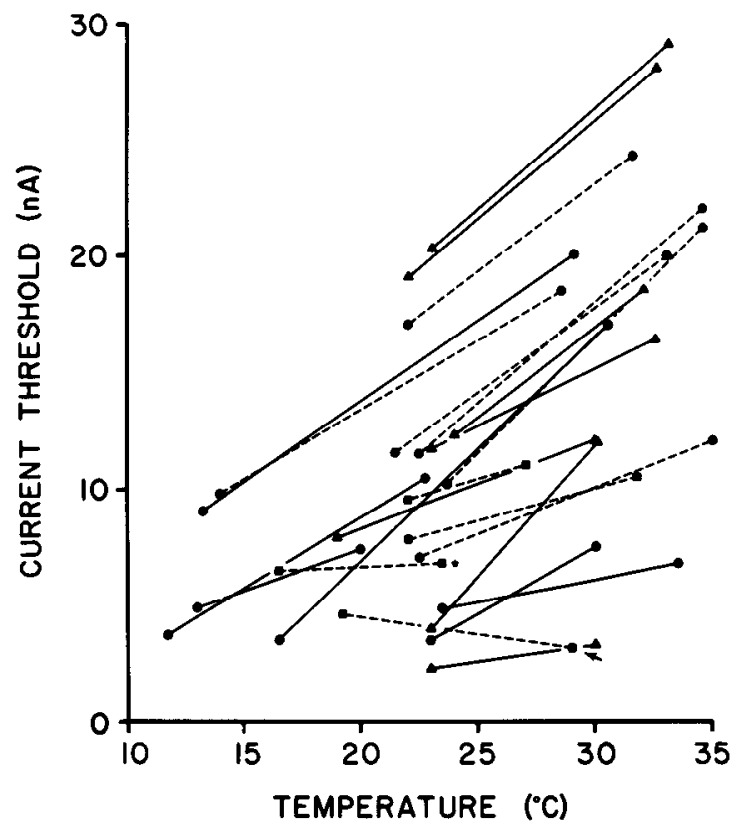

B

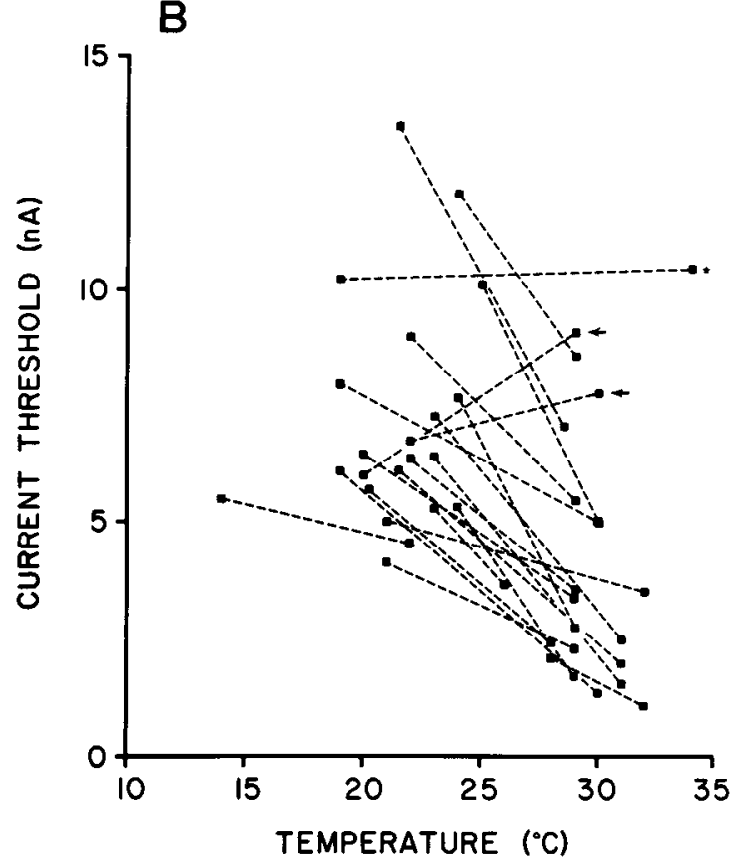

Figure 16. Variability among individual animals of the effects of temperature on the current thresholds of their FETi neurons. $A$, Results obtained in this investigation in experiments on three species of grasshoppers: $S$. americana (O), $S$. nitens $(\square)$, and $L$. migratoria $(\boldsymbol{\Lambda})$. The solid lines represent results from neuropil recordings and the dashed lines represent results from soma recordings. Threshold determinations were made with $100-\mathrm{msec}$ current pulses. $B$, Results obtained in the Heitler et al. (1977) investigation in experiments on $S$. nitens. All threshold determinations were made with soma penetrations and using 100 -msec current pulses. (The data are replotted from Goodman and Heitler, 1977, Fig. 1a.) In both $A$ and $B$, each line corresponds to a different animal. Note the variability both between and also within studies: among the 23 FETi neurons tested in this study, the typical response was an increase in the current threshold with heating; however, 1 neuron showed a decrease in threshold with heating (arrow), while, in another, the threshold remained unchanged (asterisk). Among the 22 FETi neurons examined by Heitler et al. (1977), though the usual response to heating was a decrease in the current threshold, in 2 animals, the threshold increased (arrows) and, in a 3rd, the threshold changed little (asterisk).

its FETi neuron decreased with heating rather than increasing (Fig. 16A). Thus, both types of responses to temperature may have existed in both of the $S$. nitens populations used in these studies. Secondly, genetically based variability in the physiology of identified central neurons has been demonstrated in a study of isogenic clones of $S$. americana grasshoppers by Goodman and Heitler (1977). In particular, they found differences between two clones in how the input resistance of FETi changed with temperature, corresponding to differences in the temperature dependence of FETi's voltage and current thresholds. We therefore suggest that such population differences may have been responsible for the different effects of temperature on FETi that were observed in the two studies.

There is some indication that the second delayed phase of the response of threshold to temperature changes that was observed by Heitler et al. (1977) may depend upon synaptic interactions. This second phase disappeared irreversibly following perfusion with zero $\mathrm{Ca}^{2+} / \mathrm{high} \mathrm{Mg}^{2+}$ saline. They report that, under these conditions, "an increase in temperature increased the threshold, and a decrease in temperature decreased the threshold" (Heitler et al., 1977, p. 177), exactly as in the present study. It may be that, in the majority of the $S$. nitens that they studied, a change in synaptic input to FETi occurred with heating, bringing the neuron closer to threshold.
However, there are a number of other ways in which calcium concentrations might have influenced threshold in their experiments, and we are unable to distinguish among these.

In summary, the differences between the two studies in their observations on the temperature dependence of threshold may be due to interindividual variability within the $S$. nitens populations. Should this be the case, we would expect an increase in the current threshold of central neurons with heating to be the response that is more typical of Locusta and Schistocerca since it predominated in each of the populations that we studied of three different species of these acridid grasshoppers.

\section{Frequency-current relation}

Initial firing rate. Of the various effects of temperature on central neuronal properties which we have described, the only one that would favor increased behavioral activity at higher body temperatures is the change in the slope of the f-I relation. In all interneurons and motoneurons studied, heating produced a substantial steepening of the f-I curves for the initial firing rates during current pulse injection; despite any temperature effects on the current threshold, when stimulated with sufficiently strong currents, central neurons had faster initial firing frequencies at warmer temperatures.

Steady state firing rate. In most neurons studied, 
heating also increased the slope of the f-I relation for steady state firing, although the change in slope was less than for the initial f-I relation. Thus, when these neurons were stimulated with sufficiently strong current pulses, their firing rates, both initially and after adaptation, were faster at higher temperatures.

In contrast, 2 mesothoracic interneurons, the $\mathrm{C}$ and $\mathrm{G}$ neurons, behaved differently. The slopes of their steady state f-I curves did not increase with heating but actually decreased slightly. Even when stimulated with current strengths large enough to increase their initial firing rates with heating, after adaptation, both the $\mathrm{C}$ and $\mathrm{G}$ neurons fired more slowly when warm.

\section{Synaptic transmission}

With increasing temperature, there was a reduction in the time course of the EPSPs that were studied and also a smaller and somewhat variable decrease in their amplitude. The shorter duration EPSPs at the higher temperatures would have a lower probability of summating with one another and thus would be less effective in exciting a postsynaptic neuron. However, in a warm grasshopper, any increase in the firing frequencies of presynaptic neurons due to the effect of temperature on repetitive firing would tend to compensate for the reduction in the PSP time course. Thus, it is possible that, under some circumstances, the efficacy of synaptic inputs might change relatively little with temperature.

\section{Heterogeneity among neurons in their response to temperature changes}

There was substantial variation among the different neuron types that we have studied in the way in which temperature affected neuronal properties. For example, while all motoneurons and most interneurons showed similar increases in the current threshold with heating, the majority of the $M$ interneurons in our experiments (5 out of 8 ) showed little change in threshold as a function of temperature. Similarly, the threshold of one mesothoracic dimming-sensitive interneuron showed no detectable temperature sensitivity. Such qualitative differences in the temperature sensitivities of different neurons within a single animal also have been described in cockroaches (Kerkut and Taylor, 1958) and in molluscs (Kerkut and Ridge, 1962; Willows, 1965; Murray, 1966). (In addition to qualitative differences among identified neurons, we have seen substantial quantitative variability in temperature effects. For a given identified neuron, the exact amount by which the current threshold increased with heating, the amount by which the f-I relation was steepened with heating, and the point at which warm and cool temperature f-I curves intersected one another all varied among individual grasshoppers.) One other example of qualitative variation in the effects of temperature on grasshopper neurons is discussed below.

$C$ and $G$ neurons. The $\mathrm{C}$ and $\mathrm{G}$ neurons were unique among the cells studied in that the slope of their steady state f-I relations did not increase in response to heating. It is particularly interesting that this heterogeneity in the effect of temperature on the repetitive firing properties of central neurons correlates with the developmental histories of these cells. C. Goodman and J. Raper (per- sonal communication) have shown recently that the $\mathrm{C}$ and $\mathrm{G}$ neurons originate as sibling cells during embryonic development; both are derived from the third division of an identified neuroblast (7-4) in the mesothoracic ganglion, and their initial neurites grow out from their somata in close temporal and spatial proximity. The morphological similarities between the $\mathrm{C}$ and $\mathrm{G}$ neurons are illustrated in Figure 17. Analogous similarities in the membrane properties of groups of neurons that develop over short time periods from a single neuroblast have been described by Goodman et al. (1980). In the third and perhaps also in the first thoracic ganglion, there are neurons which are homologous to the $C$ and $G$ neurons in that these cells are also the 5th and 6th neurons to develop from neuroblast 7-4 in these other ganglia. (C. Goodman, personal communication). It would be of interest to determine whether these $\mathrm{C}$ and $\mathrm{G}$ homologues also decrease their steady state firing frequencies with heating.

\section{Primary sensory neurons}

One additional result of heating was a striking increase in the responsiveness of sensory receptors to sensory stimuli. The same effects described here for auditory receptors, namely, a reduction in the threshold to sensory stimuli and an increase in responsiveness to suprathreshold stimuli, also have been seen in a variety of other types of sensory receptors (T. W. Abrams and K. G. Pearson, manuscript in preparation). In those receptors that were analyzed sufficiently, the increased responsiveness could be accounted for by an increase in the amplitude of the generator current that occurred with heating. At warmer temperatures, receptors also showed a steepening of intensity-response functions. Thus, in general, there is an increase in the sensitivity of primary sensory neurons with heating. We suggest that the resulting increase in the afferent input to the CNS may be a major factor contributing to the increased activity of central neurons at higher temperatures.

\section{Conclusion}

The two factors identified in this study that may be principally responsible for the increase in the behavioral activity of grasshoppers that occurs with warming are (1) an increase in the slope of the f-I relation and (2) an increased responsiveness to sensory stimuli in a variety of sensory receptors. These two temperature effects together with the others that we have described are classified in Table I according to whether they favor an increase or a decrease in the activity of central neurons with heating.

A major and unexpected finding of this study is that the increase in the frequency of various behaviors with warming is not accompanied by a reduction in the spike threshold of the neurons controlling those behaviors. In particular, although the frequency of jumping increases with increasing temperature, the neurons involved in producing jumping behavior show no decrease in their spike threshold. The metathoracic tibia motoneurons increased their current thresholds with heating as did the $\mathrm{C}$ neuron, the interneuron that probably initiates the preparatory co-contraction phase of the jump (Pearson 
and Robertson, 1981). The $M$ neuron, which is likely to be the interneuron that triggers the rapid extension of the fully flexed, or "cocked," tibia (Pearson et al., 1980), also showed no reduction in its threshold with heating (in some animals, the current threshold of the $\mathrm{M}$ neuron increased with heating, while in others, it remained unchanged). Thus, a decrease in the spike threshold of central neurons is not necessary for the increase in the activity of these neurons which occurs with heating.

One important conclusion suggested by Table $I$ is that it is actually the interaction of a number of temperature effects on neuronal properties which determines how the activity of an individual nerve cell will be influenced by temperature. For instance, the increases in the current threshold in response to heating will tend to be compensated by those temperature effects that favor increased activity in central neurons with increased temperature. As discussed above, the change in synaptic interactions with temperature will depend on the combined effects of decreases in PSP duration and increases in the frequency of repetitive firing in presynaptic neurons. Because the relative contribution of each of the effects listed in Table I may vary in different neuron types, different cells are likely to have different temperature sensitivities. Thus, $M$ neurons, on the average, demonstrate less change in the spike threshold with temperature than most other neurons studied.

This heterogeneity in the temperature sensitivities of neurons may be functionally important in permitting the temperature dependence of different behaviors to be independently determined. Various behaviors in grasshoppers differ in the temperature ranges at which they occur most frequently. For example, oviposition requires a higher minimum temperature than does flight (Uvarov, 1977) or feeding. We speculate that neurons that differ in their sensitivity to temperature may be involved in the control of behaviors with different temperature ranges.

The lack of a reduction in the spike threshold of the central neurons of warm grasshoppers also seems functionally adaptive when considered in a behavioral context. In contrast to what might be inferred from the

\section{TABLE I}

Factors influencing how the activity of central neurons changes with temperature

\begin{tabular}{cc}
\hline $\begin{array}{c}\text { Factors Favoring Increased Activity at } \\
\text { Warmer Temperalures }\end{array}$ & $\begin{array}{c}\text { Factors Opposing Increased } \\
\text { Activity at Warmer } \\
\text { Temperatures }\end{array}$ \\
\hline $\begin{array}{c}\text { Increased slope of initial frequency- } \\
\text { current relationship }\end{array}$ & $\begin{array}{c}\text { Increased current thresh- } \\
\text { old }\end{array}$ \\
Increased slope of steady state fre- & Decreased steady state fir- \\
quency-current relationship (most & ing rate (some interneu- \\
interneurons and motoneurons) & rons, e.g., C and G) \\
Increased afferent input; increased re- & Decreased PSP duration \\
ceptor sensitivity with reduced & (and amplitude) \\
threshold to sensory stimuli & \\
\hline
\end{tabular}

\section{C-neuron}
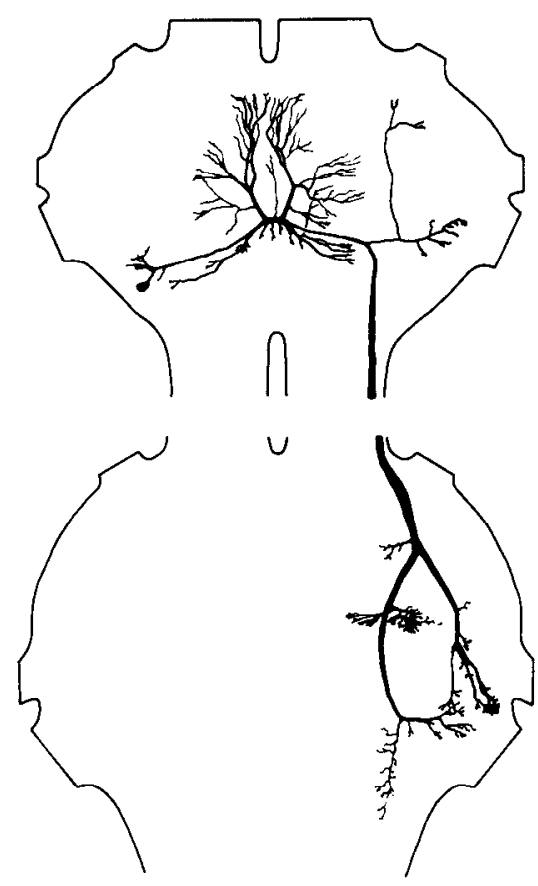

$\cup$

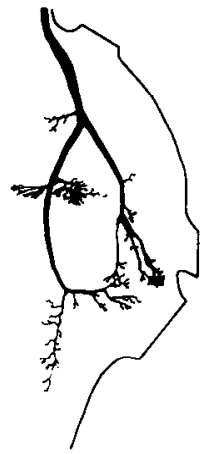

\section{G-neuron}

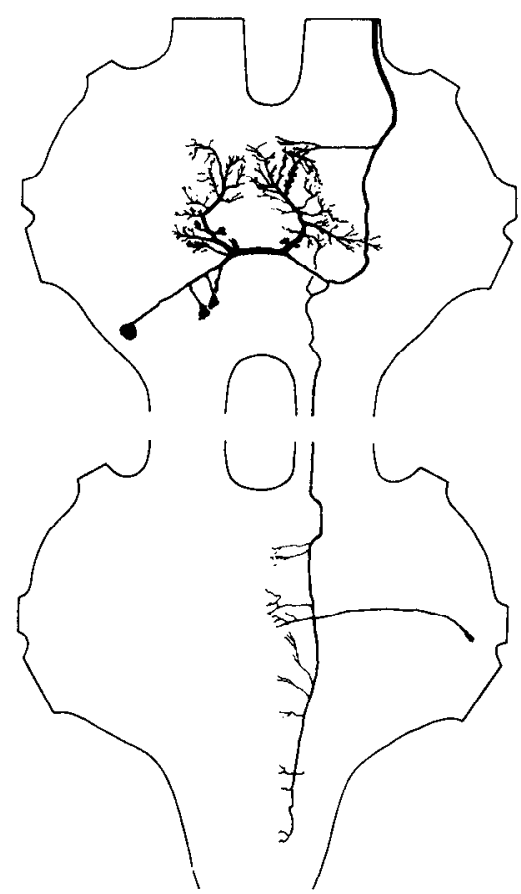

Figure 17. Morphological similarities between 2 sibling interneurons, $\mathrm{C}$ and $\mathrm{G}$. Both the G neuron and the $\mathrm{C}$ neuron have their somata located posterolaterally on the dorsal surface of mesothoracic ganglion. Both cells have their main neurites, that cross the midline and connect the bilateral halves of their dendritic fields, running in the posterior commissure of the mesothoracic ganglion. Their axonal projections differ: each posteriorly projecting axon runs in a different portion of the meso-metathoracic connective, and only the $G$ neuron sends an axon anteriorly. In addition to having similar temperature sensitivity in their steady state repetitive firing (cf., Fig. $9 B$ with Fig. $10 B$ ), the $C$ and $G$ neurons share some of the same synaptic connections; both neurons receive inputs from the DCMD, both receive auditory input, and both cells make excitatory connections with FETi (Pearson et al., 1980; Pearson and Robertson, 1981). 
introduction, grasshoppers do not become continuously active as the temperature rises. Instead, their total time spent locomoting may decrease as temperature is increased from $24^{\circ}$ to $40^{\circ} \mathrm{C}$ (Chapman, 1965). Grasshoppers may spend a maximal amount of time stationary at temperatures as high as $36^{\circ}$ to $41^{\circ} \mathrm{C}$ (Gardefors, 1964; Chapman, 1965), which therefore are known as the "preferred" ambient temperatures. In fact, these insects achieve elevated body temperatures by spending long periods basking (in our cultures, they "bask" by situating themselves adjacent to incandescent bulbs; see "Materials and Methods, Animals and rearing"). One additional example of the complexity of the relationship between temperature and behavior is that, although gregarious, grasshoppers will fly readily and for long periods once their temperature exceeds a minimum; solitary $L o$ custa and Schistocerca fly primarily at dusk or at night when their temperatures are at the low end of the range at which flight is possible (Uvarov, 1977). Given that active behaviors are not performed continuously when grasshoppers are warm, it seems consistent that there is no decrease in the threshold of the entire population of central neurons with heating. We suggest instead that, through the interaction of two opposing groups of temperature effects on neuronal properties, neuronal activity and the control of behavior are well regulated at both warm and cool temperatures.

\section{References}

Abrams, T. W. (1982) The effects of temperature on neurons and behavior in the grasshopper. Doctoral dissertation, University of Washington, Seattle.

Burrows, M., and G. Hoyle (1973) Neural mechanisms underlying behavior in the locust Schistocerca gregaria. III. Topography of the limb motorneurons in the metathoracic ganglion. J. Neurobiol. 4: 167-186.

Chapman, R. F. (1965) The behaviour of nymphs of Schistocerca gregaria (Forskal) (Orthoptera, Acrididae) in a temperature gradient with special reference to temperature preference. Behaviour 24: 283-317.

Chapman, R. F. (1969) The Insects; Structure and Function, American Elsevier, New York.

Fitzhugh, R. (1966) Theoretical effect of temperature on threshold in the Hodgkin-Huxley nerve model. J. Gen. Physiol. 49: 989-1005.

Gardefors, D. (1964) The influence of rapid temperature changes on the activity of Chorthippus albomarginatus de Geer. Entomol. Exp. Appl. 7: 71-84.

Goodman, C. S., and W. J. Heitler (1977) Isogenic locusts and genetic variability in the effects of temperature on neuronal threshold. J. Comp. Physiol. 117: 183-207.

Goodman, C. S., K. G. Pearson, and N. C. Spitzer (1980) Electrical excitability: A spectrum of properties in the progeny of a single embronic neuroblast. Proc. Natl. Acad. Sci. U.S.A. 77: 1676-1680.

Guttman, R. (1966) Temperature characteristics of excitation in space-clamped squid axons. J. Gen. Physiol. 49: 1007-1018.

Gwilliam, G. F., and M. Burrows (1980) Electrical characteristics of the membrane of an identified insect motor neurone. J. Exp. Biol. 86: 49-61.

Heitler, W. J., and M. Burrows (1977) The locust jump. II. Neural circuits of the motor programme. J. Exp. Biol. 66: 221-241.

Heitler, W. J., C. S. Goodman, and C. H. Fraser Rowell (1977) The effects of temperature on the threshold of identified neurons in the locust. J. Comp. Physiol. 117: 163-182.

Hussein, M. (1937) The effect of temperature on locust activity. Bull. Minist. Agric. Egypt Tech. Scient. Serv. No. 184.

Kalmring, K. (1975) The afferent auditory pathway in the ventral cord of Locusta migratoria (Acrididae). I. Synaptic connectivity and information processing among the auditory neurons of the ventral cord. J. Comp. Physiol. 104: 103-141.

Kerkut, G. A., and R. M. A. P. Ridge (1962) The effect of temperature changes on the activity of the neurones of the snail Helix aspersa. Comp. Biochem. Physiol. (B) 5: 283-295.

Kerkut, G. A., and B. J. R. Taylor (1958) The effect of temperature changes on the activity of poikilotherms. Behaviour 13: 259-279.

Laudien, H. (1973) Temperature and behavior. In Temperature and Life, H. Precht, J. Christopherson, H. Hensel, and W. Larcher, eds., pp. 441-453, Springer-Verlag, New York.

Laverack, M. S. (1961) The effect of temperature changes on the spontaneous nervous activity of the isolated nerve cord of Lumbricus terrestris. Comp. Biochem. Physiol. (B) 3: 136-140.

Morrissey, R., and J. S. Edwards (1979) Neural function in an alpine grylloblattid: A comparison with the house cricket, Acheta domesticus. Physiol. Entomol. 4: 241-250.

Murray, R. W. (1966) The effect of temperature on the membrane properties of neurons in the visceral ganglion of Aplysia. Comp. Biochem. Physiol. (B) 18: 291-303.

Pearson, K. G., and R. M. Robertson (1981) Interneurons coactivating hindleg flexor and extensor motoneurons in the locust. J. Comp. Physiol. 144: 391-400.

Pearson, K. G., W. J. Heitler, and J. D. Steeves (1980) Triggering of locust jump by multimodal inhibitory interneurons. $J$. Neurophysiol. 43: 257-278.

Uvarov, B. P. (1977) Grasshoppers and Locusts: A Handbook of General Acridology, Vol. 2, Centre for Overseas Pest Research, London.

Willows, A. O. D. (1965) Giant nerve cells in the ganglia of nudibranch molluses. Comp. Biochem. Physiol. (B) 14: 707-710. 